\title{
A Política Nacional de Atenção Domiciliar no Brasil: potencialidades, desafios e a valorização necessária da atenção primária à saúde
}

The National Policy for Home Care in Brazil: potentialities, challenges, and the necessary enhancement of primary health care

La Política Nacional de Atención Domiciliaria en Brasil: potenciales, desafios y la valoración necesaria de la atención primaria de salud.

Mariana Borges Dias ${ }^{1}$; Leonardo Cançado Monteiro Savassi ${ }^{2}$; Mônica Regina Macedo Toledo Prado Nunes ${ }^{3}$; Mara Lúcia Renostro Zachi ${ }^{4}$

\section{Resumo}

Em 2011, o Brasil redefiniu a Política Nacional de Atenção Domiciliar (PNAD) para o Sistema Único de Saúde, reorganizando-a em três níveis: AD1 realizada pelas equipes de Atenção Primária e AD2 e AD3, realizada por equipes de Atenção Domiciliar. O cuidado no domicílio amplia, qualifica e potencializa as experiências de cuidado, promove acessibilidade e auxilia na coordenação do cuidado de pessoas impossibilitadas de comparecer a serviços de saúde. A Atenção Domiciliar modificou o cenário das Redes de Atenção a Saúde no país, com a incorporação de equipes que podem substituir o cuidado hospitalar com qualidade, e complementar o trabalho realizado pela Atenção Primária a Saúde. São discutidas as potencialidades, as fragilidades e os desafios para a consolidação da PNAD no Brasil tendo em vista a necessidade de ampliação dessa modalidade de cuidado e consolidação da integração entre as equipes de Atenção Domiciliar e Atenção Primária a Saúde.

Descritores: Serviços de Atenção Domiciliar, Atenção Primária a Saúde, Políticas Públicas de Saúde

\section{Abstract}

In 2011, Brazil redefined the National Policy for Home Care (PNAD) for the Unified Health System, reorganizing it into three levels: AD1 carried out by primary care teams and AD2 and AD3, held by Home Care teams. The home care expands, qualifies and enhances the care experiences, promotes accessibility and assists in the coordination of care for people unable to attend health services. The Home Care changed the landscape of Care Networks Health in the country, with the incorporation of teams that can replace hospital care quality and complement the work done by the Primary Health Care. The are discussed potential, weaknesses and challenges for PNAD consolidation in Brazil in view of the need to expand this care modality and consolidation of

\footnotetext{
${ }^{1}$ Médica. Especialista em Medicina Interna. Coordenadora Geral de Atenção Domiciliar (CGAD)/ Departamento de Atenção Básica/ Ministério da Saúde. Coordenação Geral da Atenção Domiciliar (CGAD) - Edifício Premium SAF Sul - Quadra 2 - Lotes 5/6 Bloco II - Subsolo - CEP: 70.070-600 Brasília - DF. email: mariana.borges@ saude.gov.br

${ }^{2}$ Médico de Família e Comunidade. Doutor em Saúde Coletiva/ Educação em Saúde. Docente das Universidades Federais de Ouro Preto e Minas Gerais. Coordenador do Programa Multicêntrico de Qualificação em Atenção Domiciliar a distância da Universidade Aberta do SUS (UnA-SUS). Av. L3 Norte, Campus Universitário Darcy Ribeiro Gleba A, SC 04 - sala 201, CEP 70910-900 Brasília - DF. email: savassi@ medicina.ufop.br

${ }^{3}$ Terapeuta Ocupacional. Presidente da Associação Brasileira dos Serviços de Atenção Domiciliar. Coordenadora da Área de Assistência e Internação Domiciliar da Secretaria Municipal de Saúde de Campinas/SP. Av. Anchieta S/N Centro. CEP 13.015-904 Campinas - SP. email: monica.nunes@ campinas.sp.gov.br

${ }^{4}$ Enfermeira. Especialista em Vigilância Sanitária. Assessora da gestão estratégica da Secretária Municipal de Saúde de Cascavel/PR. Ex- Presidente da Associação Brasileira dos Serviços de Atenção Domiciliar. Av. Brasil, 7482 - Centro, Cascavel - PR, CEP 62.850-000. email: maraz@ cascavel.pr.gov.br
} 
ISSN 2179-6750

integration between the Home care teams and Primary Health care.

Key-words: Home Care Services, Primary Health Care, Public Health Policies

\section{Resumen}

En 2011, Brasil redefinió la Política Nacional de Atención Domiciliaria (PNAD) para el Sistema Único de Salud, la reorganización en tres niveles: AD1 llevada a cabo por equipos de atención primaria y AD2 y AD3, en poder de los equipos de cuidados en casa. La atención domiciliaria se expande, se clasifica y mejora las experiencias de cuidado, promueve la accesibilidad y la ayuda en la coordinación de la atención para las personas que no pueden asistir a los servicios de salud. El Home Care cambió el panorama de las redes de atención de salud en el país, con la incorporación de equipos que pueden reemplazar a la calidad de la atención hospitalaria y complementar el trabajo realizado por la Salud de Atención Primaria. El se discuten posibles, debilidades y desafíos para la consolidación de la PNAD de Brasil en vista de la necesidad de ampliar esta modalidad de atención y la consolidación de la integración entre los equipos de atención domiciliaria y la atención primaria de salud.

Palabras-claves: Servicios de Atención Domiciliaria, Atención Primaria de Salud, Políticas de Salud Pública

Em 2011, o Brasil redefiniu a Política Nacional de Atenção Domiciliar (PNAD), reorganizando-a em três níveis de Atenção que se complementam: o primeiro nível de Atenção Domiciliar (AD1), representado pelas equipes da Atenção Primária (APS) - sejam elas equipes de Atenção Básica (eAB) ou de Saúde da Família (eSF); e o segundo e terceiro níveis (AD2 e AD3) representado pelas equipes Multiprofissionais de Atenção Domiciliar (EMAD) e de Apoio a Atenção Domiciliar (EMAP) ${ }^{1}$.

Até então, entretanto, do ponto de vista normativo especifico, o cuidado domiciliar foi incorporado muito lentamente, necessitando treze anos entre a primeira legislação (Portaria $n^{\circ}$ 2.416, em 1998, que estabeleceu requisitos para credenciamento de hospitais e critérios para realização de internação domiciliar no SUS) e a portaria 2029, de agosto de 2011. Esta efetivamente incorporou a $\mathrm{AD}$ como novo ponto da Rede de Atenção à Saúde (RAS), destinando recursos federais de custeio mensal às equipes implantadas e tornando-se programa prioritário de governo quando foi tornou-se o Programa Melhor em Casa (PMC) em oito de novembro de $2011^{2}$.

Destacou-se ainda a Resolução normativa da Agência Nacional de Vigilância Sanitária (ANVISA) em janeiro de 2006, que dispõe sobre o funcionamento e a conceituação da AD no SUS e na Saúde suplementar. O acúmulo produzido pelas experiências municipais e hospitalares públicas em vigor nas décadas de 1990 e 2000, permitiu elaboração da regulamentação definitiva em 2011, resultando em uma proposta já pré experienciada por vários ${ }^{3}$. 
ISSN 2179-6750

Em termos de experiências brasileiras no que concerne à Atenção Domiciliar (AD), sua primeira expressão publica foi com Oswaldo Cruz e as enfermeiras visitadoras na década de 20 no século passado. Já na década de 60, o Hospital dos Servidores do Estado de São Paulo organizava um serviço próprio de AD como uma atividade planejada no setor público ${ }^{2}$.

Entretanto, foi na década de 90 que diversos municípios, inicialmente para adequarem-se ao advento do tratamento poliquimioterapico da AIDS e posteriormente como maneira de ampliar acesso e otimizar o uso do leito hospitalar. Ao fim da década de 2000, existiam várias experiências de $\mathrm{AD}$ em curso no Brasil, com diversos projetos tecnoassistenciais e que aconteciam por iniciativa das gestões municipais e de estabelecimentos hospitalares nos três níveis de governo ${ }^{4}$. Esta expansão fez com que houvesse a "necessidade de regulamentação de seu funcionamento e de políticas públicas de modo a incorporar sua oferta às práticas institucionalizadas no Sistema Único de Saúde (SUS)" 5, 6 .

O grande diferencial de eficácia da Atenção Domiciliar (AD) reside no espaço do domicilio potencializar a produção da saúde. O contexto familiar engloba questões sociais, econômicas, culturais e relacionais. Ao adentrar no espaço familiar, os profissionais inserem-se para muito além dos problemas clínicos do paciente, estando frente a frente com importantes fatores influenciadores do processo saúde-doença - o que lhes propicia uma prática bem mais desafiadora, justamente por ser abrangente, diferenciada, personalizada e humanizada. Seu objeto permanente constitui-se na atenção centrada nas necessidades do usuário, o que favorece a integralidade do cuidado ${ }^{4}$.

A AD contribui também para o cumprimento dos princípios de ampliação do acesso, acolhimento, equidade, humanização e integralidade da atenção à saúde, se inserindo nas linhas de cuidado por meio de práticas clínicas cuidadoras baseadas nas necessidades da pessoa, reduzindo a fragmentação da atenção à saúde. E adota um modelo de atenção centrado no trabalho de equipes multiprofissionais e interdisciplinares, que amplia a participação ativa da pessoa, da família, do cuidador e das equipes de saúde da Rede de Atenção a Saúde (RAS) ${ }^{7}$. Conceitualmente,

\footnotetext{
“A atenção domiciliar consiste numa modalidade de atenção à saúde substitutiva ou complementar às já existentes, caracterizada por um conjunto de ações de promoção à saúde, prevenção e tratamento de doenças e reabilitação prestadas em domicílio, com garantia de continuidade de cuidados e integrada às redes de atenção à saúde".
}

A PNAD é uma estratégia fomentada pelo PMC da Coordenação Geral de Atenção Domiciliar (CGAD) do Ministério da Saúde. Suas características peculiares de transversalidade a 
ISSN 2179-6750

diversos pontos de atenção o permitem compor formalmente a Rede de Urgências e Emergências (RUE) como componente que visa à saída qualificada das portas de urgência ${ }^{8}$.

A real inserção e consequente integração da $\mathrm{AD}$ com os demais pontos da RAS e em especial com a APS e as urgências, tem se tornado um importante desafio para os gestores. Entendese que esta integração consegue reorganizar as diversas linhas de cuidado propostas e aperfeiçoa os fluxos entre rede básica, Urgências e hospitais. Como resultado, haverá melhora na eficiência do atendimento a saúde da população.

Um dos grandes avanços da PNAD em relação às políticas anteriores reside no fato de considerar o cuidado realizado no domicílio pelas equipes de APS como parte estrutural da AD, e não como um serviço realizado à parte daquele realizado por Serviços de AD (SAD). Entendendo esta atenção como o primeiro de três níveis de atenção, inclui-se a prática das equipes de Saúde da Família e de Atenção Básica como elemento fundamental de um cuidado contínuo e complementar, somando as iniciativas destas equipes ao cuidado das EMAD e EMAP ${ }^{4}$.

Portanto, ao realizar a atenção no domicílio e deparando-se com a necessidade de um cuidado de maior densidade tecnológica ou de maior dedicação de tempo ao paciente, as equipes de APS e de AD evitam a descontinuidade deste cuidado, atuando de maneira horizontal com foco na integralidade da atenção àquela pessoa. Ao invés de se realizar um encaminhamento formal, burocrático e descontextualizado, a PNAD fomenta a troca de saberes entendendo que, sendo ambas as equipes parte do mesmo processo de trabalho, o domiciliar, a continuidade entre os níveis prevalecerá tanto na entrada do paciente no nível de atenção do SAD, quanto em seu retorno ao cuidado pela UBS ${ }^{9}$.

Além disso, a capacidade de ser substitutiva a outros pontos da rede de atenção a saúde e de desinstitucionalizar o paciente são elementos fundamentais para a produção de novas maneiras de cuidar, de novas práticas de saúde em que o compromisso com a defesa da vida norteia o pacto de trabalho das equipes. Quando a AD se configura como dispositivo substitutivo para a produção de cuidados que efetivamente não são produzidos dentro do hospital, do ambulatório ou da instituição, ela possibilita a invenção de práticas cuidadoras ${ }^{10}$.

Alguns desafios pautam os próximos passos da $\mathrm{AD}$ no Brasil. Um deles passa pela reestruturação da $\mathrm{AD} 1$, praticada pelas eAB e eSF, através do estímulo, reorganização e qualificação da APS para visitas domiciliares e AD1 adequada, integrada aos SAD. Parte deste esforço vem sendo cumprido pelas iniciativas de Educação Permanente em parceria com a UNASUS, mas tendo em vista a enorme capilaridade, diversidade de ações e funções típica de um país cuja saúde é gerida no nível do município, e qualidade técnica de seus recursos humanos, as ações 
ISSN 2179-6750

devem ser intensificadas ${ }^{11}$.

Outro envolve a potencialização das EMAD para atuação junto a serviços de urgência. A AD tem sido primordialmente uma estratégia de desospitalização de pacientes internados, ou de complementaridade aos serviços da APS, mas tem-se mostrado ainda tímida em termos de apoio a Rede de Urgência e Emergência. É preciso evidenciar para estes setores a potencia da AD como saída qualificada das portas de urgência, incluindo as portas de entrada de Hospitais, unidades de pronto-atendimento, e rede de primeiros socorros, incluso aí o SAMU ${ }^{12,13}$.

Da mesma maneira, é fundamental avançar numa política de fortalecimento da integração com os Serviços de Regulação, e mesmo na capacidade de trabalho integrado do SAD com os hospitais. O que se percebe é que embora o SAD seja parte de uma rede de cuidados, os gestores municipais ainda não conseguiram construir mecanismos de regulação que permitam a desospitalização adequada ou a oferta da $\mathrm{AD}$ como opção dentro da rede de atenção a saúde. A relação entre os serviços se fazem primordialmente via equipes de saúde, sem que mecanismos reguladores eficazes se construam para potencializar o uso da $\mathrm{AD}^{4,13,14}$.

Outro desafio fundamental a ser superado refere-se à capacidade de atuação das próprias equipes de AD. É necessário trabalhar para que elas ampliem as possibilidades de atender pacientes de maior complexidade tecnológica, através da capacitação de seus profissionais para execução de cuidados mais intensivos tais como a Ventilação Mecânica Domiciliar, maior atuação em cuidados paliativos, e ampliação do cuidado para o publico neonatal e pediátrico.

É necessário também ainda avançar na incorporação acadêmica e universitária dos preceitos da $\mathrm{AD}$, tanto em termos de uma formação voltada para este âmbito, quanto na produção de pesquisas na área. Como área de atuação ainda nova em termos de políticas públicas no Brasil, percebe-se uma insuficiência de cursos tanto na graduação quanto na pós-graduação. Soma-se a esse desafio o fato da $\mathrm{AD}$ exigir competências que não são plenamente contempladas em nenhum curso de graduação e nenhuma especialidade ou pós-graduação em saúde ${ }^{9}$.

A CGAD programa, por fim, avançar no telemonitoramento das ações dos SAD, através de um sistema de indicadores em construção ${ }^{15}$. Além disto, outras propostas de atuação da CGAD no Brasil são a de avançar em conformações possíveis para cuidados domiciliares seguros nas populações de difícil acesso, e avançar numa proposta intersetorial para o Cuidador.

\section{Referências}

1. Ministério da Saúde (BR). Portaria MS/GM nº 2.527 de 27 de outubro de 2011. Redefine a atenção domiciliar no âmbito do Sistema Único de Saúde (SUS). Diário Oficial da União, 
ISSN 2179-6750

Brasília, DF. 208, 28 out. 2011. Seção 1. p. 44.

2. Ministério da Saúde (BR). Secretaria de Atenção à Saúde. Departamento de Atenção Básica. Cadernos de Atenção Domiciliar. Brasília: Editora do Ministério da Saúde, 2012. 2 v.

3. Agência Nacional de Vigilância Sanitária. Resolução RDC nº 11, de 26 de janeiro de 2006. Dispõe sobre o Regulamento Técnico de Funcionamento de Serviços que prestam Atenção Domiciliar.

4. Neto, AVO; Dias, MB. Atenção Domiciliar no Sistema Único de Saúde (SUS): o que representou o Programa Melhor em Casa? Rio de Janeiro: Rev Divulg em Saúde p Debate 2014.(51): 58-72.

5. Silva, KL, Sena, RR, Seixas, CT, Feuerwerker, LCM, Merhy, EE. Atenção domiciliar como mudança do modelo tecnoassistencial. Revista de Saúde Pública, 2010.44(1), 166-176.

6. Ministério da Saúde (BR). Atenção domiciliar no SUS: resultados do laboratório de inovação em atenção domiciliar. Brasília: Ministério da Saúde, 2014. 184 p.

7. Ministério da Saúde (BR). Portaria MS 4.279 de 30 de dezembro de 2010. Define as Redes de Atenção à Saúde - RAS.

8. Ministério da Saúde (BR). Portaria GM/MS nº 1.601, de 7 jul 2011. Estabelece diretrizes para a implantação do componente Unidades de Pronto Atendimento (UPA 24h) e o conjunto de serviços de urgência 24 horas da Rede de Atenção às Urgências, em conformidade com a Política Nacional de Atenção às Urgências.

9. Savassi, LCM. Os atuais desafios da Atenção Domiciliar na Atenção Primária a Saúde: uma análise na perspectiva do Sistema Único de Saúde. Rev Bras Med Fam Comunid 2016. [no Prelo].

10. Feuerwerker, LCM; Merhy, EE. A contribuição da atenção domiciliar para a configuração de redes substitutivas de saúde: desinstitucionalização e transformação de práticas. Rev Panam Salud Publica 2008.24(3) 180-188.

11. Savassi, LCM; Franco, SM; Oliveira, VA. Construindo um curso a distância de atenção domiciliar multicêntrico, multiprofissional, multi-institucional, multiformatos. Gusmão, CMG; Borba, VR; Menezes-Junior, JV; Oliveira, CAP; Nascimento, EP; Oliveira, VA. II Relato de experiências em tecnologias educacionais do Sistema UNA-SUS 2015. — Recife: Ed. Universitária da UFPE, 2015. Pp 99-121.

12. Meloni, DR. Análise da implantação da atenção domiciliar no Sistema Único de Saúde [dissertação]. Ribeirão Preto (SP): Universidade de São Paulo, 2015.

13. Neto, AVO. Análise do Programa Melhor em Casa: um Olhar sobre a Atenção Domiciliar no 
ISSN 2179-6750

Âmbito do Sistema Único de Saúde [dissertação]. Brasília (DF): Universidade de Brasília, 2016.

14. Ministério da Saúde (BR). Portaria 963/2013. Redefine a Atenção Domiciliar no âmbito do Sistema Único de Saúde. Diário Oficial da União 101, 28 mai 2013. Seção 1, p. 30.

15. Ministério da Saúde (BR). Manual de Monitoramento e avaliação: Programa Melhor em Casa. 2. ed. Brasília: Editora MS, 2015. 